\title{
Review of: "Monovalent lanthanide(I) in borozene complexes"
}

\author{
You Song ${ }^{1}$ \\ 1 Nanjing University
}

Potential competing interests: The author(s) declared that no potential competing interests exist.

Molecule-based magnets have become prepossessing in molecular materials science, on account of the potential applications in information storage, spintronics, quantum computing. However, the reported molecule-based magnets are obviously not enough to apply, because the best single-molecule magnet (SMM) $\left[\left(C p^{i P r 5}\right) \operatorname{Dy}\left(C p^{*}\right)\right]^{+}$can only work below $80 \mathrm{~K}$ and are unstable.

In the process of designing SMMs, it is found that axisymmetric model plays an important role in the improvement of their working temperature, but it is not easy to find such materials from the perspective of synthesis. We cannot judge whether the blocking temperature of $\left[\left(\mathrm{Cp}^{i \operatorname{Pr} 5}\right) \operatorname{Dy}\left(\mathrm{Cp}^{*}\right)\right]^{+}$is an insurmountable limit. For the same design idea, perhaps Ln-borane (or Ln-carboborane) sandwich complexes can provide an opportunity for us to break through $80 \mathrm{~K}$. We can see this possibility from the recent work published on Nat. Commun. (2021) 12:6467 by Wan-Lu Li, Jun Li, Lai-Sheng Wang and co-workers. In their work, a concept of "borozene" was proposed, originating from benzene due to the similar electronic structure and aromaticity. Since lanthanide prefers to side-on coordinate with boron cluster to form more efficient chemical bonding, a variety of lanthanide-boron structures can be realized, such as half-sandwich, inverse sandwich, inverse multiple-decker, and metallo-borospherene type (as shown in Figure), which were verified theoretically and experimentally in Jun Li's and Lai-Sheng Wang's early works. Some structural characteristics are in line with the advantage of using axisymmetry to improve the magnetic anisotropy of SMMs. In these complexes, rare earth ions can show various oxidation states and have single electrons to determine the magnetic properties. This provides a basis and convenience for the synthesis of new molecular magnetic materials. Thus, these complexes show the possibility of increasing the blocking temperature.

At present, the largest challenge of these complexes is that they are prepared under extreme conditions, so it is hard to obtain bulky products for physical testing. However, since it is proved experimentally that such complexes can be stably synthesized in gaseous phase, it means that they may be obtained under improved conditions, further convenient for experimental testing even application. 